\title{
Tratamento endovascular de aneurisma da artéria renal por embolização com micromolas preservando o fluxo sangüíneo renal: relato de caso
}

\author{
Endovascular treatment of renal artery aneurysm using microcoil \\ embolization and renal blood flow preservation: case report
}

\author{
Marco Aurélio Cardozo ${ }^{1}$, Eduardo Lichtenfels² ${ }^{2}$ Nilon Erling Junior ${ }^{2}$, Eduardo Raupp ${ }^{5}$, \\ Dorvaldo P. Tarasconi ${ }^{3}$
}

\begin{abstract}
Resumo
O tratamento endovascular da doença aneurismática da artéria renal tem sido, cada vez mais, aceito como uma alternativa à cirurgia convencional, especialmente em casos de aneurismas complexos intra-parenquimatosos ou que comprometam a bifurcação da artéria renal. Os autores relatam a experiência do tratamento endovascular de uma paciente com aneurisma sacular da bifurcação da artéria renal direita, associado à hipertensão renovascular de difícil controle. Foi realizada a cateterização seletiva da artéria renal, com a inserção de micromolas no saco aneurismático. $\mathrm{O}$ aneurisma foi completamente ocluído com preservação total do fluxo sanguíneo renal. A evolução clínica foi satisfatória com redução significativa das medicações anti-hipertensivas. A angio-tomografia de controle, após o oitavo mês do procedimento, confirmou o sucesso do tratamento.
\end{abstract}

Palavras-chave: Embolização terapêutica, artéria renal, aneurisma.

\section{Introdução}

O tratamento cirúrgico dos aneurismas de artéria renal é, geralmente, complexo. A conduta adotada com maior freqüência é a aneurismectomia combinada com a arterioplastia ou ponte aorto-renal. A aneurismectomia pode ser realizada com sucesso em mais de $95 \%$ dos casos de aneurismas extra-parenquimatosos, enquanto o tratamento de certos tipos de aneurismas intraparenquimatosos ou que comprometem a bifurcação da artéria renal pode levar à nefrectomia parcial ou total. Recentemente, o tratamento percutâneo tem sido em-

\begin{abstract}
Endovascular treatment of renal artery aneurysmal disease has been increasingly accepted as an alternative to conventional surgery, especially in cases of renal artery bifurcation or complex intrarenal aneurysms. The authors report a case of endovascular treatment of a saccular aneurysm of the right renal artery bifurcation associated with poorly controlled renovascular hypertension. Selective catheterization of the renal artery was performed and microcoils were inserted into the aneurysmal sac. The aneurysm was completely obliterated with total preservation of renal blood flow. Clinical evolution was satisfactory with significant reduction in anti-hypertensive drugs. Control tomographic angiography, after eight months, confirmed treatment success.
\end{abstract}

Keywords: Therapeutic embolization, renal artery, aneurysm.

pregado com maior freqüência para estes casos de aneurismas complexos, evitando complicações isquêmicas renais e nefrectomias ${ }^{1,2}$.

Relatamos o caso de uma paciente em que foi realizado o tratamento endovascular por embolização com micromolas de um aneurisma sacular da bifurcação da artéria renal, com preservação completa do fluxo sanguíneo renal.

\section{Descrição do caso}

Mulher branca de 52 anos consultou em nosso serviço com quadro de hipertensão arterial de difícil

1. Professor de Cirurgia Vascular, Fundação Faculdade Federal de Ciências Médicas de Porto Aletre (FFFCMPA), Porto Alegre, RS. Cirurgião vascular e endovascular, Irmandade Santa Casa de Miresicórdia de Porto Alegre (ISCMPA), Porto Alegre, RS.

2. Residentes de Cirurgia Vascular, FFFCMPA/ISCMPA, Porto Alegre, RS.

3. Radiologistas intervencionistas, ISCMPA, Porto Alegre, RS.

Artigo submetido em 25.09.06, aceito em 22.03.07. 
controle, mesmo com o uso de quatro drogas antihipertensivas. Apresentava sobrepeso (índice de massa corporal de 28,9). Relatava início dos sintomas há 20 anos e várias tentativas de tratamento para emagrecimento, sem sucesso. Nos últimos dois anos, apresentou piora progressiva e descontrole da doença, com várias internações em emergências de hospitais, apresentando quadro de cefaléia e hipertensão arterial severa (pressão arterial variando de 200/140 $\mathrm{mmHg}$ ). A pressão arterial de base no pré-operatório era de 180/120 mmHg (medida pela manhã, três dias diferentes, esfigmomanômetro Tycos $\left.{ }^{\circledR}\right)$. Medicamentos em uso: atenolol $200 \mathrm{mg} /$ dia, enalapril $40 \mathrm{mg} / \mathrm{dia}$, hidroclortiazida $25 \mathrm{mg} / \mathrm{dia}$, nifedipina $40 \mathrm{mg} / \mathrm{dia}$. A cintilografia renal com tecnécio associado a captopril evidenciou hipertensão renovascular relacionada ao rim direito. A angio-tomografia demonstrou aneurisma sacular de $8 \mathrm{~mm}$ de diâmetro na bifurcação da artéria renal direita (Figura 1). Apresentava creatinina sérica de $0,7 \mathrm{mg} / \mathrm{dL}$.

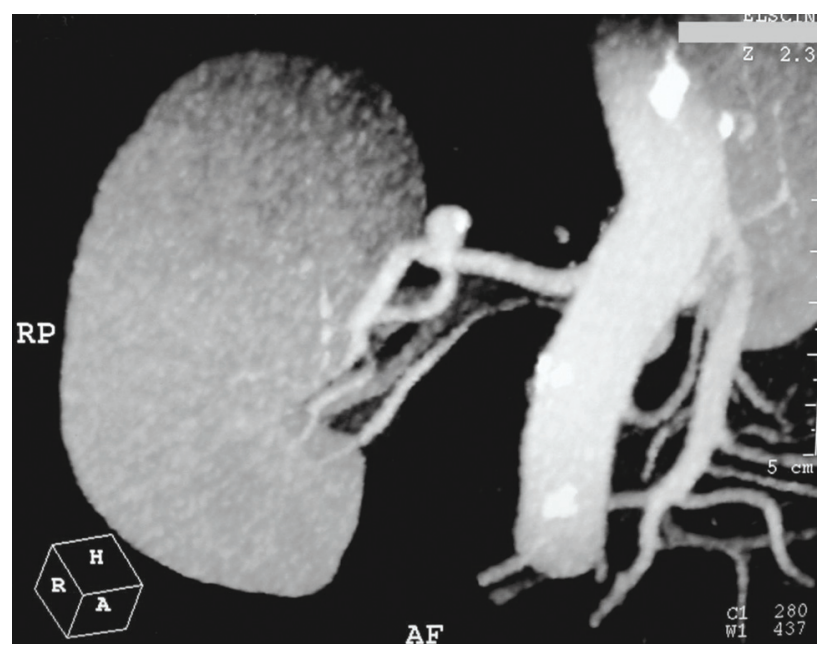

Figura 1 - Angio-tomografia pré-operatória demonstrando aneurisma sacular da bifurcação da artéria renal direita

A decisão pela intervenção baseou-se na presença de aneurisma sacular da artéria renal associado a hipertensão renovascular de difícil controle do rim ipsilateral. O procedimento endovascular foi realizado sob anestesia local. Foi inserido um introdutor $5 \mathrm{~F}$ na artéria femoral comum direita e realizada heparinização sistêmica. Através de um cateter Cobra II de 5F (Cordis), utilizado para cateterização seletiva da artéria renal direita, foi inserida, dentro do saco aneurismático, uma microguia Transcend 0,10 e um microcateter Excelsior 1048 (Boston Scientific). Via microcateter, foram liberadas, seqüencialmente, sete molas destacáveis Guglielmi (GDC $^{\mathrm{TM}}$ Boston Scientific) dentro do aneurisma (Figura 2). Foi realizada uma arteriografia de controle que demonstrou obliteração completa do aneurisma, preservando o fluxo sanguíneo pela artéria renal (Figura 3). A paciente teve excelente recuperação pósoperatória e recebeu alta no segundo dia após o procedimento. Foi indicado o uso de ácido acetil-salićlico e clopidogrel durante 6 semanas. A pressão arterial em repouso no pós-operatório era de 140/100 $\mathrm{mmHg}$. Medicações utilizadas para controle da pressão arterial no pós-operatório: atenolol $100 \mathrm{mg} / \mathrm{dia}$, hidroclortiazida $25 \mathrm{mg} / \mathrm{dia}$, amlodipina $10 \mathrm{mg} / \mathrm{dia}$. A angiotomografia de controle, realizada no oitavo mês de seguimento, demonstrou oclusão total do aneurisma e preservação da circulação renal distal.

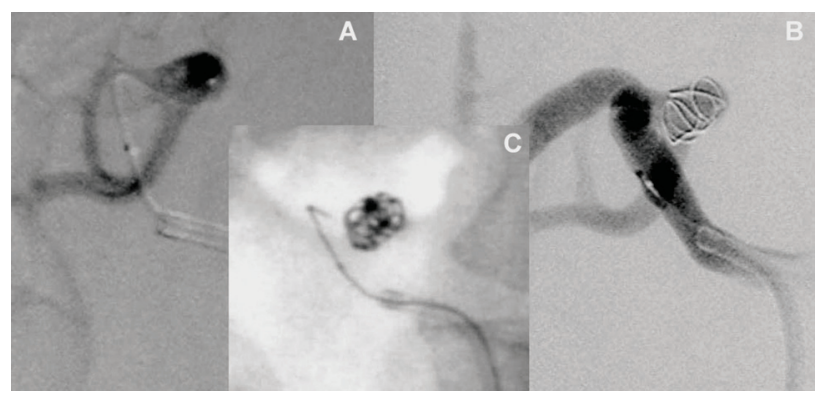

Figura 2 - A: cateterização seletiva do aneurisma; B e C: embolização do saco aneurismático com micromolas

\section{Discussão}

$\mathrm{O}$ aneurisma da artéria renal representa uma patologia pouco usual. Normalmente, se apresentam como um achado incidental em estudos de imagem realizados para a investigação de outras doenças, especialmente hipertensão. Existe uma associação entre estas duas 


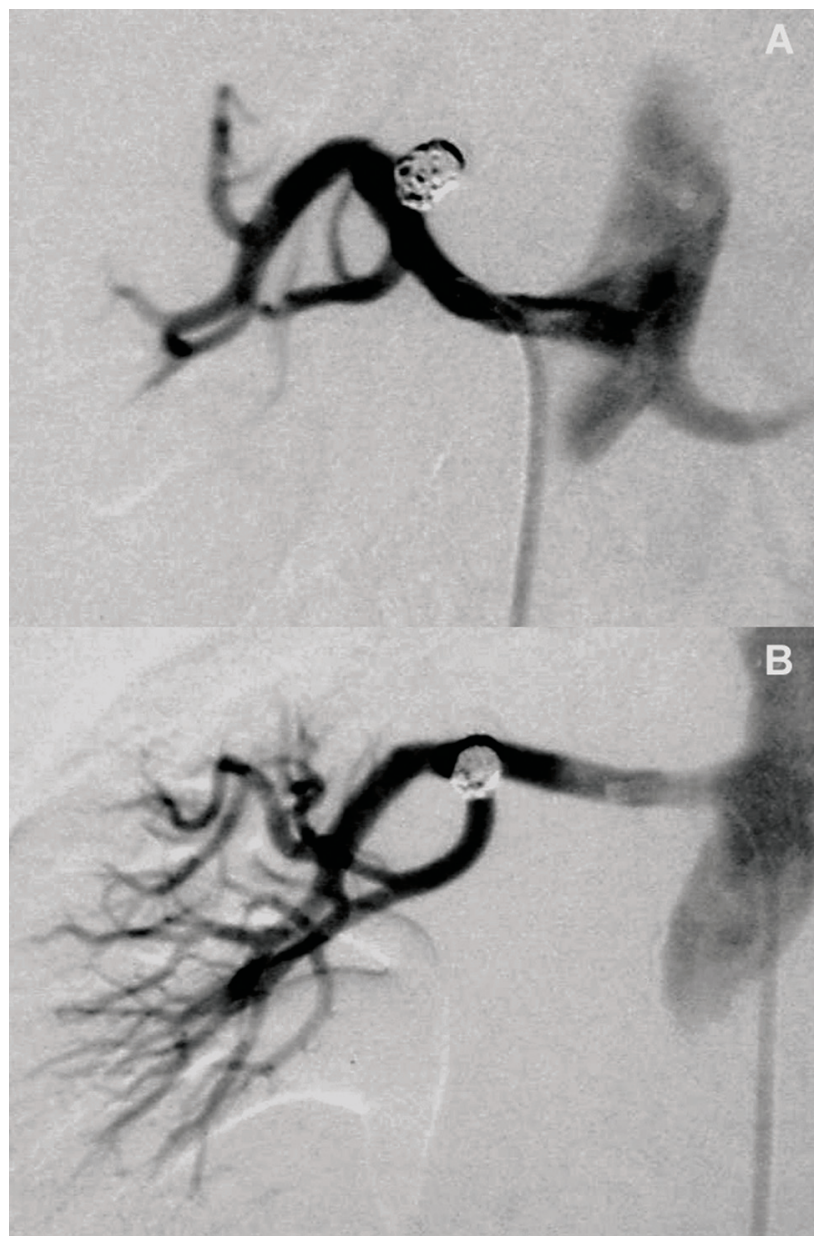

Figura 3 - Angiografia final demonstrando oclusão do saco aneurismático com preservação do fluxo sanguíneo pela artéria renal

patologias em $70 \%$ dos $\operatorname{casos}^{3}$, mas apenas alguns apresentam hipertensão de origem renovascular ${ }^{4}$. Diversas razões são sugeridas para este efeito, entre elas: embolização, compressão extrínseca e tortuosidade da artéria adjacente ${ }^{5}$. Embora o mecanismo exato para as alterações de fluxo ainda seja assunto de debate, tem-se obtido melhora do controle da pressão arterial após a ressecção de aneurismas da artéria renal em pacientes que demonstram estudos funcionais com lado afetado ipsilateral ao do aneurisma ${ }^{6}$.

Freqüentemente, $20 \mathrm{~mm}$ de diâmetro tem sido considerado o parâmetro, em relação ao tamanho, para a indicação do tratamento. Por outro lado, têm sido relatados casos com ruptura de aneurismas com diâmetros inferiores ${ }^{6,7}$. O tipo fusiforme e as calcificações da parede arterial sugerem proteção contra a ruptura. No entanto, diversas séries não têm demonstrado a correlação entre estas características e o risco de ruptura ${ }^{4,8-11}$. O tamanho do aneurisma como parâmetro exclusivo para a indicação do tratamento não deve ser a regra, devendo ser considerado dentro de um contexto que leve em conta a idade do paciente, a presença de sintomas e a hipertensão severa associada, como no caso apresentado. Não é recomendado o reparo de aneurismas da artéria renal menores do que $20 \mathrm{~mm}$ de diâmetro em adultos que não apresentem sintomas e/ou hipertensão arterial renovascular severa associados ${ }^{12}$.

Devido ao fato de os aneurismas da artéria renal estarem usualmente localizados próximos da bifurcação da artéria renal principal ${ }^{3,5}$, a oclusão inadvertida da artéria renal em procedimento cirúrgico convencional ou implante de stent revestido (endoprótese) podem levar a infarto significativo do parênquima renal.

Com o desenvolvimento de sistemas de microcateteres e microguias, projetados inicialmente para a neurorradiologia intervencionista ${ }^{13}$, os aneurismas saculares da artéria renal podem ser excluídos seletivamente, sem comprometer o suprimento sanguíneo para os diversos segmentos do parênquima renal. As micromolas permitem uma liberação precisa após a confirmação da correta posição da mola através da arteriografia. Essa técnica reduz o risco de migração da mola, apresentando melhor resultado imediato ${ }^{14}$. A presença de um colo longo e estreito é um achado anatômico que auxilia na prevenção do deslocamento inadvertido das molas ${ }^{1,15}$. Apesar disso, aneurismas com colo largo têm sido tratados com igual sucesso ${ }^{2}$.

O resultado desse tratamento a longo prazo ainda não está bem estabelecido, e apesar da melhora no controle da pressão arterial após a exclusão endovascular do aneurisma da artéria renal, não sabemos se ele continuará se expandindo, podendo levar à recidiva dos sintomas. Dessa forma, indicamos o tratamento para pacientes sintomáticos (dor e hematúria), mulheres em período fértil, aneurisma do tipo sacular e hipertensão 
renovascular grave associada. Consideramos, portanto, a terapêutica endovascular com embolização através de micromolas uma alternativa possível para o tratamento de aneurisma da artéria renal, como demonstrado neste caso relatado.

\section{Referências}

1. Karkos CD, D'Souza SP, Thomson GJ, Chomal A, Matanhelia SS. Renal artery aneurysm: endovascular treatment by coil embolisation with preservation of renal blood flow. Eur J Vasc Endovasc Surg. 2000;19:214-6.

2. Dib M, Sedat J, Raffaelli C, Petit I, Robertson WG, Jaeger P. Endovascular treatment of a wide-neck renal artery bifurcation aneurysm. J Vasc Interv Radiol. 2003;14:1461-4.

3. Henke PK, Cardneau JD, Welling TH 3rd, et al. Renal artery aneurysms: a 35-year clinical experience with 252 aneurysms in 168 patients. Ann Surg. 2001;234:454-62.

4. Tham G, Ekelund L, Herrlin K, Lindstedt EL, Olin T, Bergentz SE. Renal artery aneurysms. Natural history and prognosis. Ann Surg. 1983;197:348-52.

5. Soussou ID, Starr DS, Lawrie GM, Morris GC Jr. Renal artery aneurysm. Long-term relief of renovascular hypertension by in situ operative correction. Arch Surg. 1979; 114:1410-5.

6. Martin RS 3rd, Meacham PW, Ditesheim JA, Mulherin JL Jr., Edwards WH. Renal artery aneurysm: selective treatment for hypertension and prevention of rupture. J Vasc Surg. 1989;9:26-34.

7. Hidai H, Kinoshita Y, Murayama T, et al. Rupture of renal artery aneurysm. Eur Urol. 1985;11:249-53.

8. Eskandari MK, Resnick SA. Aneurysms of the renal artery. Semin Vasc Surg. 2005;18:202-8.
9. Reiher L, Grabitz K, Sandmann W. Reconstruction for renal artery aneurysm and its effect on hypertension. Eur J Vasc Endovasc Surg. 2000;20:454-6.

10. Mercier C, Piquet P, Piligian F, Ferdani M. Aneurysm of the renal artery and its branches. Ann Vasc Surg. 1986;1:321-7.

11. Stanley JC. Natural history of renal artery stenosis and aneurysm. In: Calligaro KD, Dougherty MJ, Dean RH, editors. Modern management of renovascular hypertension and renal salvage. Baltimore: Williams \& Wilkins; 1996. p. $15-45$.

12. English WP, Pearce JD, Craven TE, et al. Surgical management of renal artery aneurysms. J Vasc Surg. 2004;40:53-60.

13. Murayama Y, Vinuela F, Duckwiler GR, Gobin YP, Guglielmi G. Embolization of incidental cerebral aneurysms by using the Guglielmi detachable coil system. J Neurosurg. 1999;90:207-14.

14. Klein GE, Szolar DH, Breinl E, Raith J, Schreyer HH. Endovascular treatment of renal artery aneurysms with conventional non-detachable microcoils and Guglielmi detachable coils. Br J Urol. 1997;79:852-60.

15. Routh WD, Keller FS, Gross GM. Transcatheter thrombosis of a leaking saccular aneurysm of the main renal artery with preservation of renal blood flow. AJR Am J Roentgenol. 1990;154:1097-9.
Correspondência:
Marco Aurélio Cardozo
Rua Marquês do Pombal, 1199/401
CEP 90540-001 - Porto Alegre, RS
Tel.: (51) 3337.2306
E-mail: macardozo@terra.com.br 